\title{
Robust Backstepping Tracking Control of Mobile Robot Based on Nonlinear Disturbance Observer
}

\author{
Mahmood Ali Moqbel Obaid****, Abdul Rashid Husain*, Ali Abdo Mohammed Al-kubati** \\ * Faculty of Electrical Engineering, Universiti Teknologi Malaysia, Malaysia \\ ** Faculty of Computer Science and Engineering, Hodeidah University, Yemen
}

\section{Article Info \\ Article history: \\ Received Sep 27, 2015 \\ Revised Dec 24, 2015 \\ Accepted Jan 16, 2015}

\section{Keyword:}

Bounded backstepping control Fuzzy logic control Nonlinear disturbance observer Tracking control Wheeled mobile robot

\begin{abstract}
This paper presents a robust backstepping control (BC) methodbased on nonlinear disturbance observer (NDOB) for trajectory tracking of the nonholonomic wheeled mobile robot (WMR) in the presence of external disturbances and parameters uncertainties. At first, a bounded Fuzzy logic based backstepping controller (BFLBC) is designed to control the WMR without considering the effects of the external disturbances and the parameters uncertainties. Typically, the conventional BC controller depends upon the state tracking errors analysis, where unbounded velocity signal is produced for the applications that have huge tracking errors. Therefore, a fuzzy logic controller (FLC) is introduced in this research in order to normalize the state tracking errors, so that the input errors to the $\mathrm{BC}$ are bounded to a finite interval. Finally, the designed BFLBC is integrated with the nonlinear disturbance observer in order to attenuate the external disturbances and model uncertainties. The simulation results show the effectiveness of the proposed controller to generate a bounded velocity signal as well as to stabilize the tracking errors to zero. In addition, the results prove that the proposed controller provide an excellent disturbance attenuation as well as robustness against the parameters uncertainties.
\end{abstract}

Copyright (c) 2016 Institute of Advanced Engineering and Science. All rights reserved.

\section{Corresponding Author:}

Mahmood Ali Moqbel Obaid, Faculty of Electrical Engineering, Universiti Teknologi Malaysia, Email: eng_mahkah192@yahoo.com

\section{INTRODUCTION}

The research on trajectory tracking problem of wheeled mobile robot (WMR) gains a great interest in the recent years due to its promising applications in many fields such as factory automation, transportation, room cleaning, security and space exploration. The main motivations behind these considerable interests are the unexpected growth in the areas of wireless communication, computing machinery and sensors technology. The purpose of the path tracking controller is to force the WMR to achieve a desired path such that the tracking error is stabilized to zero. However, the tracking error is mostly unavoidable since the performance of the WMR can be affected by different types of uncertainties such as sensors and actuators faults, slippage, friction and unmodeled dynamics. Thus, design of robust path tracking controller for WMR is still an open issue in robotics community [1].

The path tracking problem of WMR currently receives a lot of attention from both academics and automobile industrial researches. The available WMR tracking approaches have been reported in the literature can be classified into backsteeping [1-7], sliding mode [8-9], linearization [10], neural network [1112] and fuzzy logic control [13]. Although the extensive research has been done on WMR tracking control, but most of the previous work [1-10] has assumed that the WMR has a pure non-holonomic constraint. However, the pure rolling and non-slipping assumptions cannot be guaranteed due to a set of factors that influence the performance of the WMR such as sharp turning motion at high speed [13]. To overcome these 
limitations, some researchers have proposed a tracking controller for the WMR with non ideal nonholonomic constraints [13-14]. For instance, a fuzzy logic controller is proposed in [13] for tracking control of the WMR in the presence of kinematic and dynamic uncertainties, which the kinematic disturbance is assumed to be a function of both linear as well as angular velocities.

Among the previous control techniques, the backstepping controller (BC) is the most popular method for nonholonomic WMR tracking control [1-6]. In particular, the kinematic based BC approach for a nonholonomic WMR was first suggested by Kanayama et al. (1990), which it has been assumed that the WMR has a pure nonholonomic constraint. Nevertheless, a new challenging will be imposed in the controller design by violating the ideal nonholonomic constraints in which the controller should incorporates the disturbance removal to achieve high tracking accuracy. Therefore, in this paper, a non linear disturbance observer (NDOB) is combined with the $\mathrm{BC}$ controller in order to improve the disturbances attenuations and the robustness against the parameters uncertainties. Essentially, the NDOB has been applied in the literature for many nonlinear systems, such as missiles [15-17], robot manipulator [18-20] and pendubot system [21].

This research proposed a robust $\mathrm{BC}$ for uncertain kinematic model of the nonholonomic WMR based on NDOB. The proposed controller includes two parts. First, a bounded Fuzzy logic based backstepping controller (BFLBC) is designed to control the WMR without considering the effects of the external disturbances and the parameters uncertainties. Meanwhile, the fuzzy logic controller (FLC) is integrated with the $\mathrm{BC}$ to overcome the unrealistic large velocity signal that produced by the BC caused by the huge state tracking errors. Therefore, it can achieve a smooth motion of the WMR without any sharp speed jumps even for the systems that have large state tracking errors. Finally, the designed BFLBC is integrated with the NDOB in order to tackle the external disturbances and model uncertainties.

The paper is outlined as follows: In section 2, the methods are presented, which include the model of the nonholonomic WMR with kinematic uncertainties, the controller design, and the stability analysis. The simulation setup is given in section 3. The results of the proposed controller as compared to the conventional $\mathrm{BC}$ are discussed in section 4. Finally, the conclusion is presented in section 5.

\section{RESEARCH METHOD}

\subsection{Nonholonomic WMR Model with Kinematic Uncertainties}

The nonholonomic WMR model in a 2-D Cartesian workspace is shown in Figure 1, where $\{X, O$, $Y\}$ and $\{D, C, L\}$ are the global and local Cartesian coordinate system, respectively. $D$ is the driving direction (longitudinal direction) and $L$ is the lateral direction (latitudinal direction). A posture of a nonholonomic WMR in the global Cartesian coordinate system $\{X, O, Y\}$ can be represented by a vector $P_{c}=\left[X_{c} Y_{c} \Theta_{c}\right]^{T}$, where $C$ is the center of the robot, $\left(X_{c}, Y_{c}\right)$ is the WMR coordinate and $\theta_{c}$ is the orientation angle of the WMR.

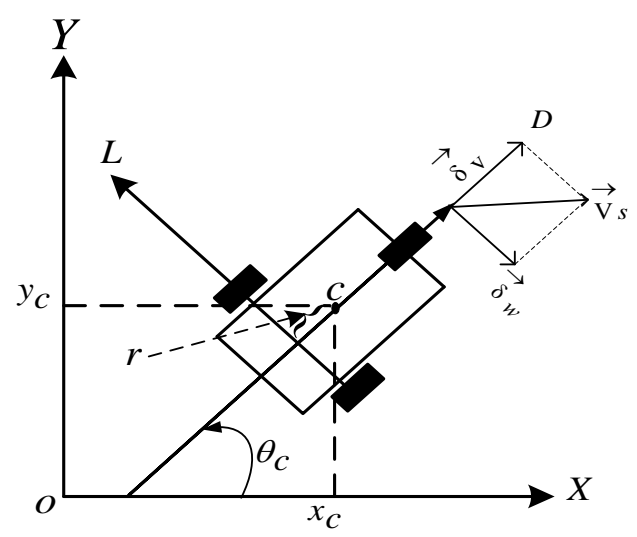

Figure 1. WMR model with kinematics uncertainties

The kinematics model of the nonholonomic WMR can be written as: 


$$
\left[\begin{array}{c}
\dot{x}_{c} \\
\dot{y}_{c} \\
\dot{\theta}_{c}
\end{array}\right]=\left[\begin{array}{cc}
\cos \theta_{c} & -\mathrm{r} \sin \theta_{c} \\
\sin \theta_{c} & \mathrm{r} \cos \theta_{c} \\
0 & 1
\end{array}\right]\left[\begin{array}{c}
v_{c} \\
w_{c}
\end{array}\right]+\left[\begin{array}{c}
\delta_{x} \\
\delta_{y} \\
0
\end{array}\right]
$$

where $v_{c}, w_{c}$ and $r$ represent the forward velocity ,angular velocity and the distance from the configuration center of the WMR to the wheels center, respectively. Moreover, $\delta_{x}$ and $\delta_{y}$ representing the kinematics uncertainties in the $D$ and $L$ coordinates, respectively. Based on study [13], the equations of the kinematic uncertainties are given as follows:

$$
\begin{aligned}
& \delta_{x}=\delta_{v} \cos \theta_{c}+\delta_{w} \sin \theta_{c} \\
& \delta_{y}=\delta_{v} \sin \theta_{c}-\delta_{w} \cos \theta_{c}
\end{aligned}
$$

where,

$$
\delta_{v}=\tanh \left(v_{c}\right), \delta_{w}=0.2 \tanh \left(w_{c}\right)
$$

\subsection{Bounded Fuzzy Logic Based Backstepping Controller Design}

The purpose of WMR path tracking controller is to find a control low input $\mathrm{q}\left(\mathrm{q}=\left[\begin{array}{lll}\mathrm{v}_{\mathrm{c}} & \mathrm{w}_{\mathrm{c}}\end{array}\right]^{\mathrm{T}}\right)$ that force the robot to follow reference trajectory with position $\left[\begin{array}{lll}x_{d} & y_{d} & \theta_{d}\end{array}\right]^{T}$ and velocity $\left[\begin{array}{ll}v_{d} & w_{d}\end{array}\right]^{T}$, such that the posture error $E_{q}\left(E_{q}=\left[\begin{array}{lll}e_{D} & e_{L} & e_{\Theta}\end{array}\right]^{T}\right)$ converges to zero as the time approaches infinite. The conventional $\mathrm{BC}$ method for tracking control of the WMR kinematic model is given by [1]

$$
\left[\begin{array}{c}
v \\
w
\end{array}\right]=\left[\begin{array}{c}
k_{1} e_{D}+v_{d} \cos e_{\Theta} \\
w_{d}+k_{2} v_{d} e_{L}+k_{3} v_{d} \sin e_{\theta}
\end{array}\right]
$$

The BC approach is limited for the applications that have small tracking errors since the controller is directly related to the state tracking errors. The FLC combined with the BC approach to overcome this limitation of the BC method. Firstly, the FLC is utilized to normalize the tracking error in the longitudinal direction $e_{D}$. Then, the BC approach is applied to control the motion of the WMR based on the normalized tracking errors, which are obtained from the FLC.The BFLBC for the kinematic model of the WMR without considering the effects of the external disturbances and the parameters uncertainties is given as follows:

$$
\left[\begin{array}{c}
v_{c 1} \\
w_{c 1}
\end{array}\right]=\left[\begin{array}{c}
k_{D}\left(1-e^{\frac{-t}{T}}\right) e_{D n o r m}+v_{r} \cos e_{\Theta} \\
w_{r}+k_{L} v_{r} e_{L}+k_{\Theta} \sin e_{\Theta}
\end{array}\right]
$$

where $v_{r}$ is the desired forward velocity, $w_{r}$ the desired angular velocity, $e_{D n o r m}$ is the normalized error in the longitudinal direction which is obtained by the FLC and T is the time constant.

\subsection{Nonlinear Disturbance Observer Design}

A NDOB is developed in this section to estimate the WMR kinematic uncertainties. Then, the NDOB is integrated with the proposed BFLBC to construct the controllerof the WMR. The kinematics model of the nonholonomic WMR can be rewritten as:

$$
\dot{\xi}=g(x) u+d
$$

where $\dot{\xi}=\left[\begin{array}{l}\dot{x}_{c} \\ \dot{y}_{c}\end{array}\right], g(x)=\left[\begin{array}{cc}\cos \theta_{c} & -\mathrm{r} \sin \theta_{c} \\ \sin \theta_{c} & \mathrm{r} \cos \theta_{c}\end{array}\right], u=\left[\begin{array}{c}v_{c} \\ w_{c}\end{array}\right], d=\left[\begin{array}{l}\delta_{x} \\ \delta_{y}\end{array}\right]$

Throughout this paper, the robot kinematic uncertainties are assumed to be slowly time varying. Motivated by the work proposed in [15-20], the following observer is designed to estimate the unknown kinematic uncertaintiesd, given by

$$
\begin{aligned}
& \hat{d}=z+p(x) \\
& \dot{z}=-l z-l(p(x)+g(x) u)
\end{aligned}
$$


where $\hat{d}, z$ and $l$ represent the estimated disturbance, the observer internal state, and the observer gain, respectively. The observer gain $l$ is given as follows:

$$
l=\frac{\partial p(x)}{\partial x}
$$

wherep $(x)=\xi$

\subsection{Backstepping Controller Design Based on Nonlinear Disturbance Observer}

In this section, the composite controller law is designed for the uncertain kinematic model WMR. The proposed controller is composed of the BFLBC as given in equation (6) and the NDOB as designed in the previous section. The composite control low $u=\left[\begin{array}{ll}v_{c} & w_{c}\end{array}\right]^{\prime}$ is given as follows:

$$
u=\eta(x)+\mu(x) \hat{d}
$$

where $\eta(x)$ is the feedback BFLBC for the WMR without considering the effects of the uncertainties, $\mu(x)$ is the disturbance compensation gain and $\hat{d}$ is the estimated disturbance using the proposed NDOB.

$$
\left[\begin{array}{l}
\eta_{11}(x) \\
\eta_{21}(x)
\end{array}\right]=\left[\begin{array}{c}
v_{c 1} \\
w_{c 1}
\end{array}\right]=\left[\begin{array}{c}
k_{D}\left(1-e^{\frac{-t}{T}}\right) e_{D n o r m}+v_{r} \cos e_{\Theta} \\
w_{r}+k_{L} v_{r} e_{L}+k_{\Theta} \sin e_{\theta}
\end{array}\right]
$$

The disturbance compensation gain $\mu(x)$ is given as follows:

$$
\begin{aligned}
& \mu(x)=-g(x)^{-1} \mathrm{C} \\
& g(x)=\left[\begin{array}{cc}
\cos \theta_{c} & -\mathrm{r} \sin \theta_{c} \\
\sin \theta_{c} & \mathrm{r} \cos \theta_{c}
\end{array}\right], \mathrm{c}=\left[\begin{array}{cc}
\mathrm{c}_{\mathrm{x}} & 0 \\
0 & \mathrm{c}_{\mathrm{y}}
\end{array}\right]
\end{aligned}
$$

wherec $c_{x}$ and $c_{y}$ are the controller gain to be design.

\subsection{Stability Analysis}

\section{Theorem 1:}

If the controller (6) is applied to the WMR kinematic model given in (1), $\boldsymbol{E}_{p}=0$ is a stable equilibrium point.

Proof:

A lyapunov function candidate $V$ is chosen as follows;

$$
V=\frac{1}{2}\left(e_{D}^{2}+e_{L}^{2}\right)+\frac{1}{k_{L}}\left(1-\cos _{\theta}\right)
$$

Clearly, $V \geq 0$ and $V=0$ if $e_{D}=0, e_{L}=0$ and $e_{\theta}=0$

$$
\begin{aligned}
& \dot{V}=e_{D} \dot{e_{D}}+e_{L} \dot{e_{L}}+\frac{\sin e_{\theta}}{k_{L}} \dot{e_{\theta}} \\
& \dot{V}=e_{D}\left[w_{c} e_{L}-v_{c}+v_{r} \cos e_{\theta}\right]+e_{L}\left[-w_{c} e_{D}+v_{r} \sin e_{\theta}\right]+\frac{\sin e_{\theta}}{k_{L}}\left[w_{r}-w_{c}\right] \\
& \dot{V}=e_{D}\left[-\left(k_{D}\left(1-e^{\frac{-t}{T}}\right) e_{D}+v_{r} \cos e_{\theta}\right)+v_{r} \cos e_{\theta}\right]+e_{L}\left[v_{r} \sin e_{\theta}\right]+\frac{\sin e_{\theta}}{k_{L}}\left[w_{r}-w_{c}\right] \\
& \dot{V}=-k_{D}\left(1-e^{\frac{-t}{T}}\right) e_{D}^{2}+e_{L}\left[v_{r} \sin e_{\theta}\right]+\frac{\sin e_{\theta}}{k_{L}}\left[w_{r}-\left[w_{r}+k_{L} v_{r} e_{L}+k_{\theta} \sin e_{\theta}\right]\right] \\
& \dot{V}=-k_{D}\left(1-e^{\frac{-t}{T}}\right) e_{D}^{2}-\frac{k_{\theta}}{k_{L}} \sin e_{\theta}{ }^{2} \leq 0
\end{aligned}
$$


Therefore $\dot{V}$ is negative semi-definite and the resulting system is asymptotically stable.

\section{SIMULATION SETUP}

The proposed controllerand the kinematic model of the robot as defined in equation (1) are simulated in MATLAB-SIMULINK. The simulation interval is from 0 to 40 second, and the sampling timet is 0.01 second. The input parameters to the system are as follows: $r=1, k_{1}=4, k_{2}=2.5, k_{3}=1, v_{d}=1$, $w_{d}=1, k_{D}=3, k_{L}=1, k_{\Theta}=1.5, C_{x}=1.3, C_{y}=1.3$ and $T=1$.Moreover, the NDOB gain is selected as follow:

$$
l=\left[\begin{array}{cc}
28 & 0 \\
0 & 28
\end{array}\right]
$$

\section{RESULTS AND ANALYSIS}

This section presents two case studies that will illustrate the robustness and the effectiveness of the proposed controller to tackle the external disturbances and model uncertainties. For the first trajectory tracking case, the proposed controller is applied to track a simple straight path with a constant linear and angular velocity. Then, a circular path is set to be a reference trajectory. Finally, the disturbance rejection ability of the proposed controller is compared with the conventional BC approach.

\subsection{Straight Path Tracking}

Initially, a simple case study to track a straight path is proposed. The desired straight path is defined as $y=10$ and $x=t+10$ in the Cartesian coordinates workspace. The initial posture of the WMR is $(0,0,0)$, while the desired initial posture is $(10,10,0)$. Thus the initial tracking error is $(10,10,0)$. Figures $2-3$ show the performances of straight path trajectory using the proposed controller. It can be observed from Figure 2 that the proposed controller generates continuous, bounded and smooth control signals with zero value at the starting time. The posture error of the straight path trajectory is shown in Figure 3. As it can be seen, the proposed controller stabilizes the tracking error to zero.

\subsection{Circular Path Tracking}

In this section, a circular path is set to be a reference trajectory. The simulation interval is from 0 to 40 second, and the sampling time $t$ is 0.01 second. The initial posture of the WMR is $(0,0.6,0)$, while the desired initial posture is $(15,13,0)$. Therefore, the initial tracking error is $(15,13,0)$. The desired circular path in the Cartesian coordinates is defined as follows:

$$
x=15-2 \cos \left(t+\frac{\pi}{2}\right), y=15-2 \sin \left(t+\frac{\pi}{2}\right)
$$

Figures 4-5 show the circular path tracking performance of the proposed controller and the standard BC method, respectively. In comparison with the conventional $\mathrm{BC}$ approach, the simulation results prove that the tracking performance can be improved significantly using the proposed controller, where it is capable to track a circular path accurately even in the presence of external disturbances and parameters uncertainties. Figures 6-7 show the velocities response of the proposed controller and the conventional BC, respectively. It can be seen form Figure 6 that the proposed controller is capable of producing a bounded as well as smooth velocity signal with zero value at the starting time. However, as shown in Figure 7 the forward velocity of the conventional $\mathrm{BC}$ jumps to more than $70 \mathrm{~m} / \mathrm{s}$ at the initial time, which is difficult to be implementable in an actual robotic system. The posture error of the proposed controller and the the conventional BC are shown in Figures 8 and 9, respectively. It can be observed that the normalizing error using FLC is converged to the natural error as the time approach infinity. In addition, the proposed controller is stabilized the tracking errors to zero. However, as shown in Figure 9, the conventional BC is failed to stabilize the tracking error to zero. Figures 10-11 show the estimation of the disturbances and parameters uncertainties using the NDOB. It can be observed that the proposed controller has an excellent disturbance attenuation as well as strong robustness against the parameters uncertainties. 


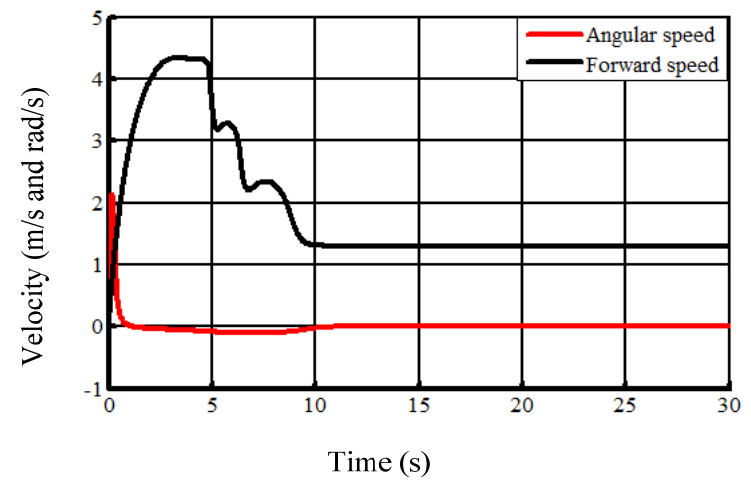

Figure 2. Control signals

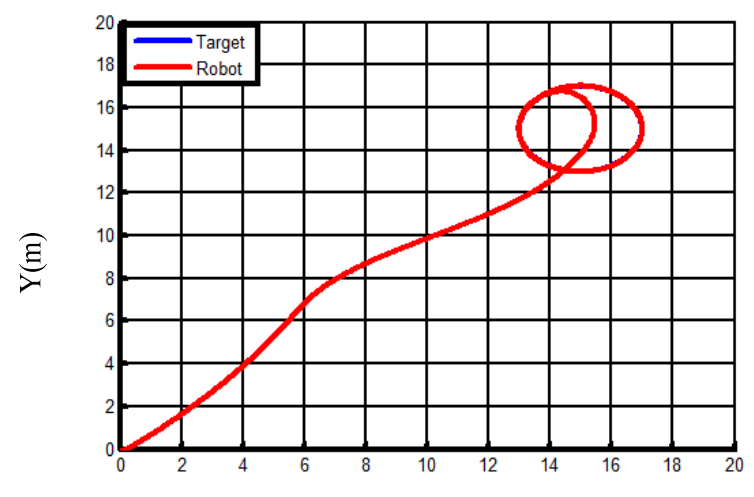

$\mathrm{X}(\mathrm{m})$

Figure 4. Trajectory of circle tracking for the proposed controller

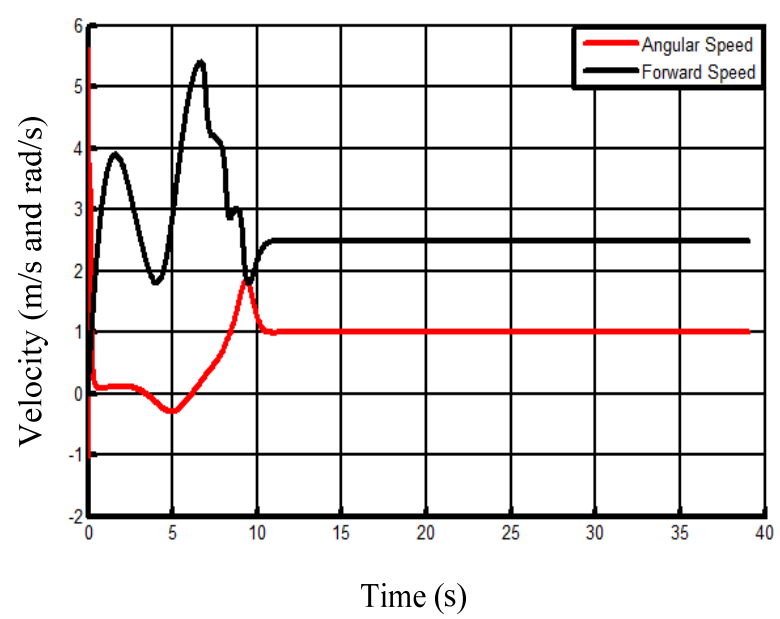

Figure 6. Control signals for the proposed controller

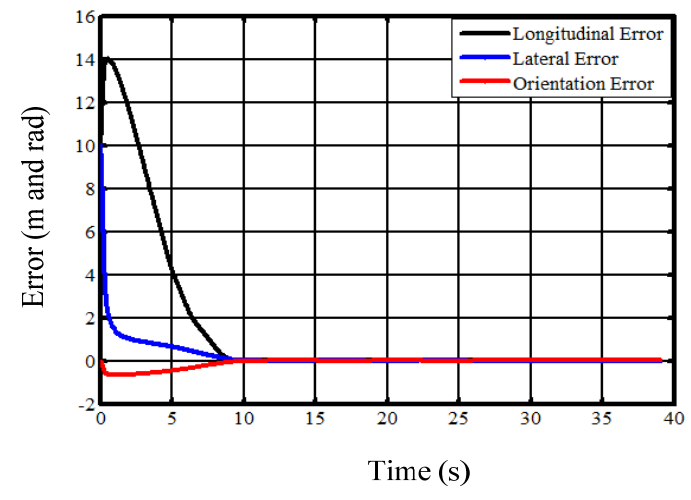

Figure 3. Posture error

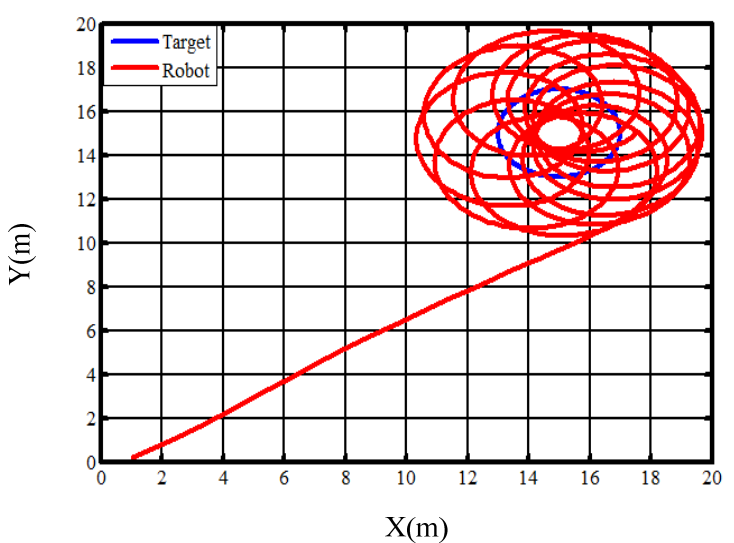

Figure 5. Trajectory of circle tracking for standard backstepping controller

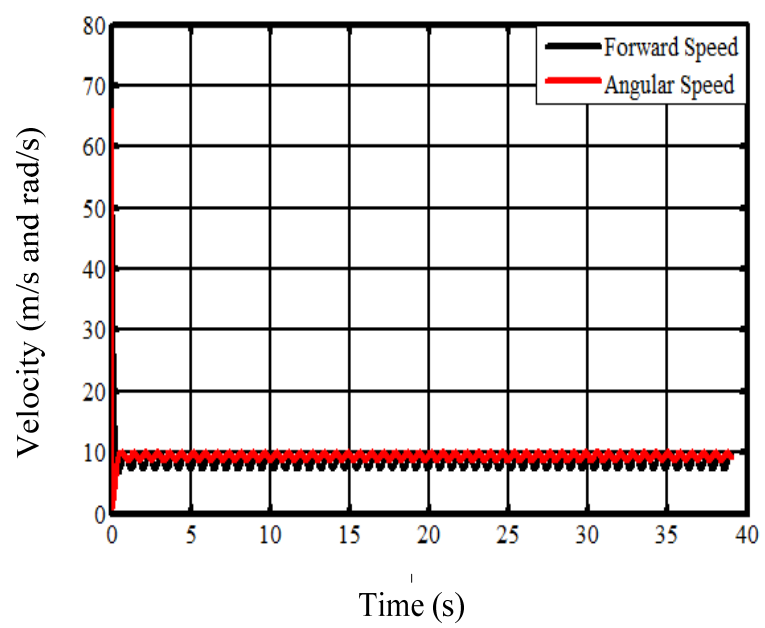

Figure 7. Control signals for the standard backstepping controller 


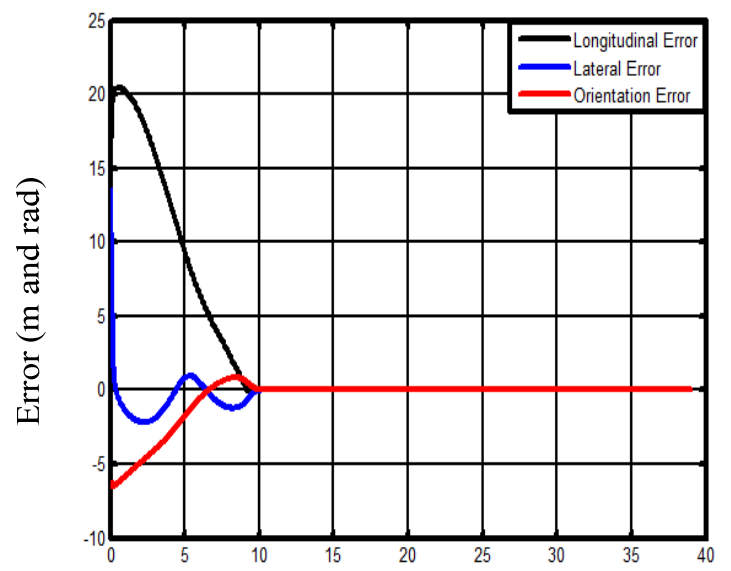

Time (s)

Figure 8. Posture error for the proposed controller

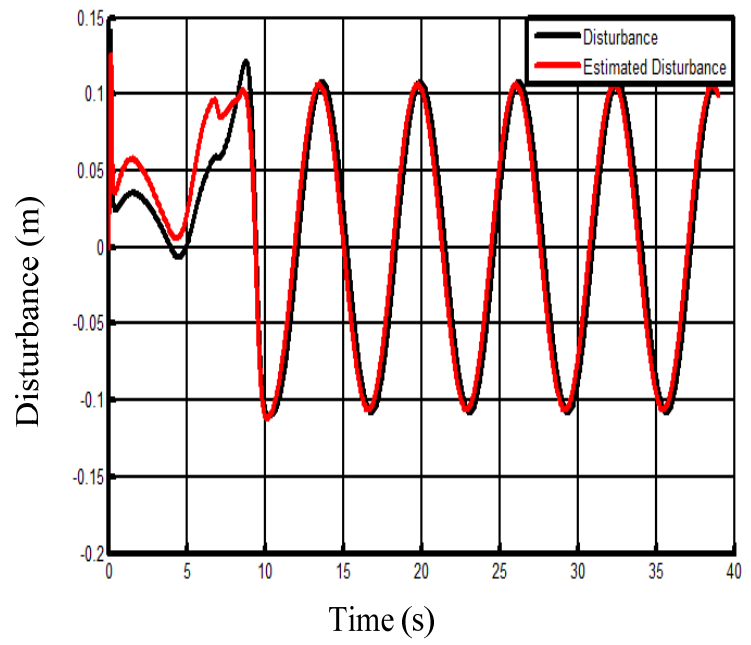

Figure 10. Disturbance estimation in the D coordinate using NDOB.

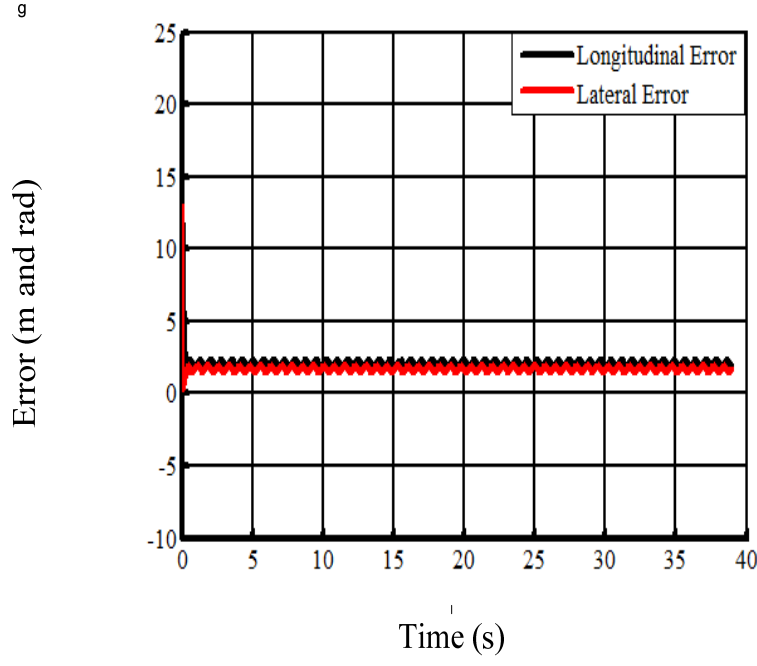

Figure 9. Posture error for the standard backstepping controller

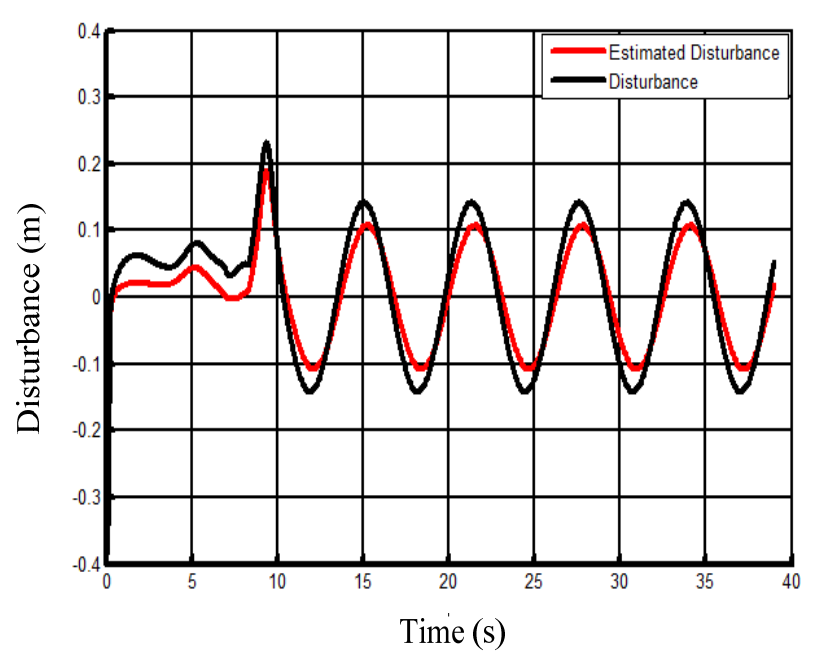

Figure 11. Disturbance estimation in the L coordinate using NDOB.

\section{CONCLUSION}

A robust $\mathrm{BC}$ controller based on nonlinear disturbance observer is presented in this research for tracking control of the kinematic model of the nonholonomic WMR in the presence of external disturbances and parameters uncertainties. The proposedcontroller is capableofproducing a bounded velocity signals regardless of the amount of the state tracking errors. Therefore, it can achieve a smooth motion for the WMR even for the applications that have huge state tracking errors. In addition, the simulation results has shown that the proposed controller provide an excellent disturbance attenuation as well as strong robustness against the parameters uncertainties.

\section{REFERENCES}

[1] S.X. Yang, A. Zhu, G. Yuan, and M.Q.H. Meng, (2012). A bioinspired neurodynamics-based approach to tracking control of mobile robots. IEEE Trans. Ind. Electron., 59(8), 3211-3220.

[2] Y. Kanayama, Y. Kimura, F. Miyazaki, T. Noguchi, (1990). A stable tracking control method for an autonomous mobile robot. In Proc. IEEE Int. Conf. Robot. Automat, 384-389. 
[3] I.M.H. Sanhoury, S.H.M. Amin, A.R. Husain, (2011).Trajectory tracking of steering system mobile robot. 4th International Conference on Mechatronics (ICOM), 1-5.

[4] R. Fierro, F.L. Lewis, (1997). Control of a nonholomic mobile robot: backstepping kinematics into dynamics. Journal of Robotic Systems, 149-163.

[5] Chwa, D. (2010). Tracking control of differential-drive wheeled mobile robots using a backstepping-like feedback linearization.IEEE Transactions on Systems, Man and Cybernetics, Part A: Systems and Humans, 40(6), 12851295.

[6] Obaid, M.A.M. and Husain, A.R. (2015). Time varying backstepping control for trajectory tracking of mobile robot. Int. J. Computational Vision and Robotics, Vol. X, No. Y, pp. 000-000.

[7] Qiao, W.Y. (2013). Backstepping Adaptive Fuzzy Scheme for SCARA GRB400 Robot. TELKOMNIKA Indonesian Journal of Electrical Engineering, 11(8), 4229-4237.

[8] D. Chwa, (2004).Sliding-mode tracking control of nonholonomic wheeled mobile robots in polar coordinates.IEEE Trans. Control Syst. Technol., 12(4), 637-644.

[9] G.G. Rigatos, C.S. Tzafestas, and S.G. Tzafestas, (2000). Mobile robot motion control in partially unknown environments using a sliding-mode fuzzy logic controller. Robot. Auton. Syst., 33(1), 1-11.

[10] D.H. Kim and J.H. Oh, (1999). Tracking control of a two-wheeled mobile robot using input-output linearization.Control Eng. Practice, 7(3), 369-373.

[11] Mohareri, O., Dhaouadi, R., \& Rad, A.B. (2012). Indirect adaptive tracking control of a nonholonomic mobile robot via neural networks. Neurocomputing, 88, 54-66.

[12] Yan-dong, L., Ling, Z., \& Ming, S. (2013). Adaptive RBFNN Formation Control of Multi-mobile Robots with Actuator Dynamics. TELKOMNIKA Indonesian Journal of Electrical Engineering, 11(4), 1797-1806.

[13] Chwa, D. (2012). Fuzzy adaptive tracking control of wheeled mobile robots with state-dependent kinematic and dynamic disturbances. IEEE Transactions on Fuzzy Systems, 20(3), 587-593.

[14] Taheri Kalani, J., \& Khosrowjerdi, M.J. (2014). Adaptive trajectory tracking control of wheeled mobile robots with disturbance observer. International Journal of Adaptive Control and Signal Processing, 28(1), 14-27.

[15] Chen, W.H. (2004). Disturbance observer based control for nonlinear systems.IEEE/ASME Transactions on Mechatronics, 9(4), 706-710.

[16] Chen, W.H. (2003). Nonlinear disturbance observer-enhanced dynamic inversion control of missiles. Journal of Guidance, Control, and Dynamics, 26(1), 161-166.

[17] Yang, J., Chen, W.H., \& Li, S. (2011). Non-linear disturbance observer-based robust control for systems with mismatched disturbances/uncertainties. IET control theory \& applications, 5(18), 2053-2062.

[18] Chen, W.H., Ballance, D.J., Gawthrop, P.J., \& O'Reilly, J. (2000). A nonlinear disturbance observer for robotic manipulators. IEEE Transactions on Industrial Electronics, 47(4), 932-938.

[19] Mohammadi, A., Tavakoli, M., Marquez, H.J., \&Hashemzadeh, F. (2013). Nonlinear disturbance observer design for robotic manipulators. Control Engineering Practice, 21(3), 253-267.

[20] Nikoobin, A., \& Haghighi, R. (2009). Lyapunov-based nonlinear disturbance observer for serial n-link robot manipulators. Journal of Intelligent and Robotic Systems, 55(2-3), 135-153.

[21] Eom, M., \& Chwa, D. (2015). Robust Swing-Up and Balancing Control Using a Nonlinear Disturbance Observer for the Pendubot System with Dynamic Friction. IEEE Transactions on Robotics, 31(2), 331-343. 